\title{
Signature of Hot Phonons in Reliability of Nitride Transistors and Signal Delay
}

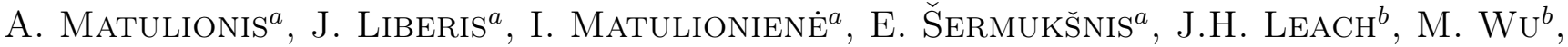 \\ X. $\mathrm{NI}^{b}$ AND H. MORKOÇ \\ ${ }^{a}$ Semiconductor Physics Institute, Centre for Physical Sciences and Technology, Vilnius, Lithuania \\ ${ }^{b}$ Department of Electrical and Computer Engineering, Virginia Commonwealth University, Richmond, VA, USA
}

Lifetime of non-equilibrium (hot) phonons in biased GaN heterostructures with two-dimensional electron gas channels was estimated from hot-electron fluctuations. Dependence of the lifetime on the electron density is not monotonous - the resonance-type fastest decay serves as a signature of hot phonons. The signature is resolved in nitride heterostructure field effect transistors when the gate voltage is used to change the channel electron density. The transistor cut-off frequency decreases on both sides of the resonance in agreement with the enhanced electron scattering caused by longer hot-phonon lifetimes. The signature is also noted in device reliability experiment: the enhanced temperature of hot phonons, possibly, triggers formation of new defects and accelerates device degradation.

PACS: $72.70 .+\mathrm{m}, 73.50 . \mathrm{Fq}, 73.61 . \mathrm{Ey}$

\section{Introduction}

Nominally undoped nitride heterostructure field effect transistors (HFETs) demonstrate excellent power performance at microwave frequencies [1]. Device degradation and rapid decrease of power generation efficiency at millimetre-wave frequencies are among the main drawbacks [2].

A high density of electrons is a prerequisite for power operation. Simultaneously, a faster operation is expected, unless electron scattering increases with the electron density. The active component of an HFET is a two-dimensional electron gas (2DEG) located in the undoped GaN layer where the dominant electron scattering by optical phonons assumes no dependence on electron density if the phonons reside at equilibrium.

The Fröhlich interaction is strong in GaN, and, as such, longitudinal optical (LO) phonons, rather than acoustic phonons, dominate hot-electron scattering at strong electric fields. Non-equilibrium (hot) LO phonons accumulate due to combined action of LO-phonon emission/ reabsorption by the electrons and conversion of the hot phonons into other phonon modes [3]. Furthermore, the physical removal of hot phonons from the channel is hampered because of the low group velocity of LO phonons. Under steady state, the emission nearly balances the absorption, and the conversion determines the net result $[3,4]$. Hot-phonon lifetime is often used to quantify the conversion [1-5].

According to optical pump-probe Raman measurements for bulk GaN, the hot-phonon lifetime decreases from $\approx 2.5 \mathrm{ps}$ at a carrier density of $10^{16} \mathrm{~cm}^{-3}$ down to 350 fs at $\approx 2 \times 10^{19} \mathrm{~cm}^{-3}$ (Fig. 1, squares [6]). Correspondingly, the hot-phonon accumulation tends to decrease. At a low carrier density, the conversion of hot phonons into acoustic phonons is assisted by transverse optical phonons [5]. The decrease of the lifetime at higher densities (squares) has been associated with plasmon-assisted conversion of hot phonons into migrant phonon modes (dashed curve [7]).

The strongest plasmon-LO-phonon interaction takes place at an electron density of $\approx 10^{19} \mathrm{~cm}^{-3}$ when the plasmon energy approaches the LO-phonon energy in GaN. Typical nitride power HFETs seek to exploit higher electron densities. Our main goal is to discuss hot-phonon effects on degradation and frequency performance of GaN-based HFETs. The discussion is supported by hot-phonon lifetime data at electron densities beyond the values reached by the Raman experiments.

\section{Hot-phonon signature: fluctuations}

The fluctuation technique [8] was applied to GaN-based 2DEG channels and yielded the lifetime in a wide range of electron sheet densities $[3,4,9]$. The non-monotonous dependence was revealed at low electric fields (Fig. 1, triangles and circles). The pioneering result (down-triangle [8]) was reproduced through the time-resolved optical pump-probe LO-phonon-assisted intersubband absorption experiment [10] carried out for a similar 2DEG channel (up-triangle). The triangles are consistent with the LO-phonon-plasmon model (dashed curve [7]) if the average electron density per unit volume is estimated as the 2DEG density divided by the channel width at the Fermi energy.

The lifetime reaches a minimum value at some resonance electron density $n_{\text {res }}$. The experimental results of Fig. 1 can be fitted with the simple resonance expression (solid line) [11]:

$$
\tau=a\left[1+\frac{b}{\left(\sqrt{n}-\sqrt{n}_{\mathrm{res}}\right)^{2}+c}\right]^{-1},
$$

where $a, b$, and $c$ are the fitting constants and $n$ is the 


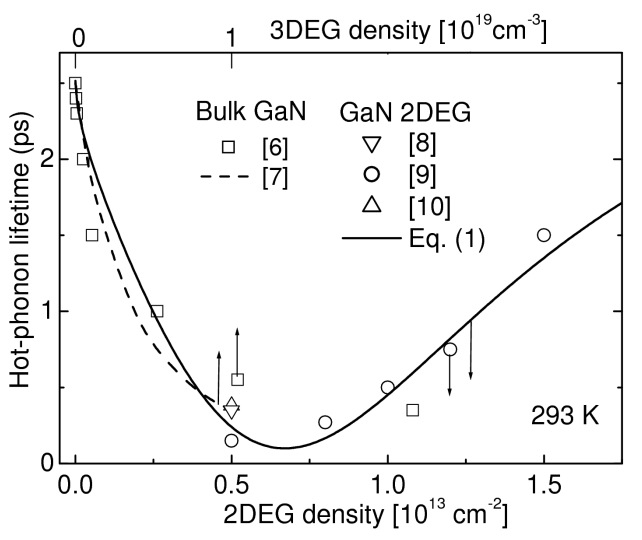

Fig. 1. Resonance dependence of hot-phonon lifetime on carrier density at low electric field. Squares Raman data (top scale) [6], circles and down-triangle - fluctuation technique (bottom scale) $[4,8,9]$, up-triangle - intersubband absorption (bottom) [10], dashed curve - plasmon-LO-phonon model (top) [7], solid curve - Eq. (1) (bottom).

2DEG density. The fitting for the 2DEG channels located in GaN yields the resonance density $n_{\mathrm{res}} \approx 7 \times 10^{12} \mathrm{~cm}^{-2}$ (Fig. 1, solid curve) [11]. In agreement with the resonant plasmon-assisted decay, the resonance density is lower in arsenide 2DEG channels $\left(n_{\text {res }} \approx 2.3 \times 10^{12} \mathrm{~cm}^{-2}[4]\right)$. The resonance described by Eq. (1) is a signature of hot phonons and plasmons useful for resolving the hot-phonon effects in HFET performance.

\section{Power-tuned resonance}

In voltage-biased 2DEG channels, hot-electron temperature depends on supplied electric power. This has an effect on plasma frequency because of gas expansion under heating. The peak electron density per unit volume decreases as the temperature increases [3]. As a result, the resonance $2 \mathrm{DEG}$ density $n_{\text {res }}$ increases with the hot-electron temperature (Fig. 2, circles) [12].

The resonance 2DEG density has been determined from the hot-phonon lifetime dependence on the hot-electron temperature available from experiment on the 2DEG channel confined in an InAlN/AlN/GaN heterostructure [3]. The estimated slope of the increase makes $2.7 \times 10^{9} \mathrm{~cm}^{-2} \mathrm{~K}^{-1}$ (Fig. 2, line). A similar value for the slope follows from the self-consistent SchrödingerPoisson calculations of the electron density profile at different electron temperatures [12].

The data of Fig. 2 illustrate that the resonance takes place at $\approx 7 \times 10^{12} \mathrm{~cm}^{-2}$ at room temperature, but $n_{\text {res }}=$ $1 \times 10^{13} \mathrm{~cm}^{-2}$ at a hot-electron temperature of $\approx 1500 \mathrm{~K}$, and $n_{\text {res }}=1.4 \times 10^{13} \mathrm{~cm}^{-2}$ at $\approx 3000 \mathrm{~K}$. Thus, if the 2DEG density in the channel exceeds the resonance value at low electric fields ( $\left.n>n_{\text {res }}\right)$, the channel can be tuned into the resonance through heating of the electron gas [9].

The ultrafast decay of hot phonons causes a lower occupancy of the hot-phonon modes and weaker hot-phonon

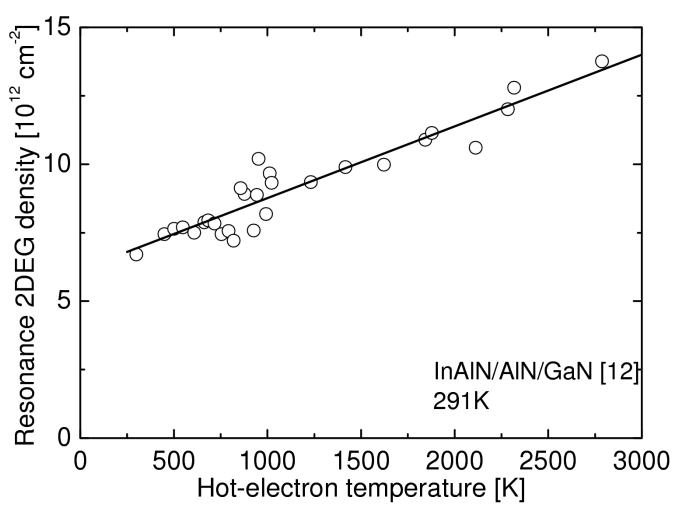

Fig. 2. Dependence of estimated resonance 2DEG density on hot-electron temperature (circles [12]).

effects at the resonance density [4]. HFETs allow one to change the electron density with the gate voltage while the drain voltage can be kept constant in order to maintain the same electric power supplied to an average electron. In this framework, the associated minima of hot phonon effects can serve as a signature of hot phonons in HFET performance.

\section{Hot-phonon signature: HFET reliability}

Device reliability was investigated on a set of nominally identical InAlN/AlN/GaN HFETs prepared from the same wafer $[2,13,14]$. Drain current was measured as a function of time for the devices biased to the same drain voltage of $20 \mathrm{~V}$ and different gate voltages. Circles in Fig. 3 illustrate the relative degradation of the drain current after the same amount of charge of $500 \mathrm{C}$ has passed through the HFET channels that contained different 2DEG density under the gate determined by the gate voltage selected in the range from $-3 \mathrm{~V}$ to $-7 \mathrm{~V}$.

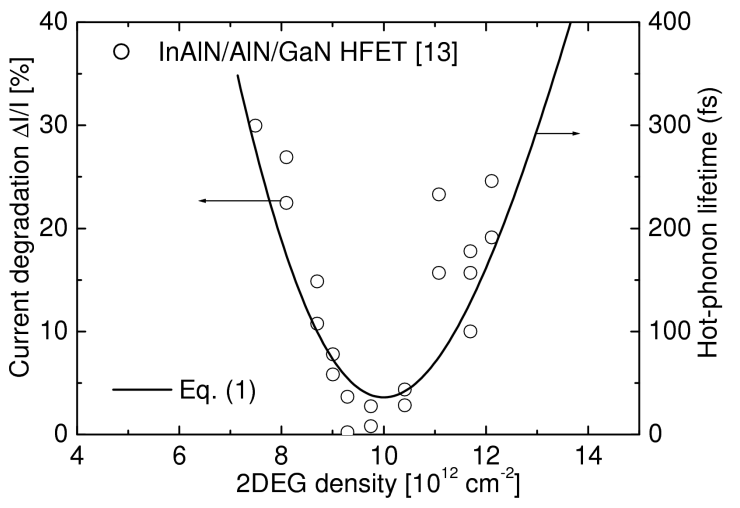

Fig. 3. Current degradation of $\mathrm{InAlN} / \mathrm{AlN} / \mathrm{GaN}$ HFETs (circles [13]) correlates with hot-phonon lifetime illustrated with Eq. (1) for $n_{\text {res }}=1 \times 10^{13} \mathrm{~cm}^{-2}$ (curve).

The non-monotonous density-dependent current degradation is difficult to interpret if hot phonons 
are ignored. A strong correlation of the hot-phonon lifetime and the HFET degradation is evidenced through comparison of circles and solid curve in Fig. 3. A longer lifetime on both sides of the resonance (curve) means a higher occupancy of the hot-phonon modes, and the associated enhanced ionic vibrations cause a higher probability for defect formation. As mentioned, the resonance $2 \mathrm{DEG}$ density of $1 \times 10^{13} \mathrm{~cm}^{-2}$ (curve) is reached at the hot-electron temperature of $\approx 1500 \mathrm{~K}$ (Fig. 2).

\section{Hot-phonon signature: signal delay}

High occupancy of LO phonon modes enhances probability of electron scattering. The probability of LO-phonon emission increases in proportion to $N+1$ while the probability of reabsorption increases in proportion to $N$, where $N$ is the occupancy. When the emission follows the reabsorption immediately, the electron energy remains almost conserved, but the direction of motion changes. This has an impact on electron velocity as shown through Monte Carlo simulation [8]. As discussed, the hot-phonon effect is stronger if the lifetime is longer.

Experiments on HFETs yield the electron drift velocity through measurement of the intrinsic transit time needed for an electron to traverse the high-field region located under the gate in the channel. The results for an InAlN/ AlN/GaN HFET were obtained from the signal delay time extracted from the unity-gain cut-off frequency [15] (Fig. 4, circles). The shortest delay is reached near the 2DEG density of $9.5 \times 10^{12} \mathrm{~cm}^{-2}$. The experimental data are fitted with Eq. (1) when $n_{\text {res }}=9.5 \times 10^{12} \mathrm{~cm}^{-2}$ is assumed (solid curve).

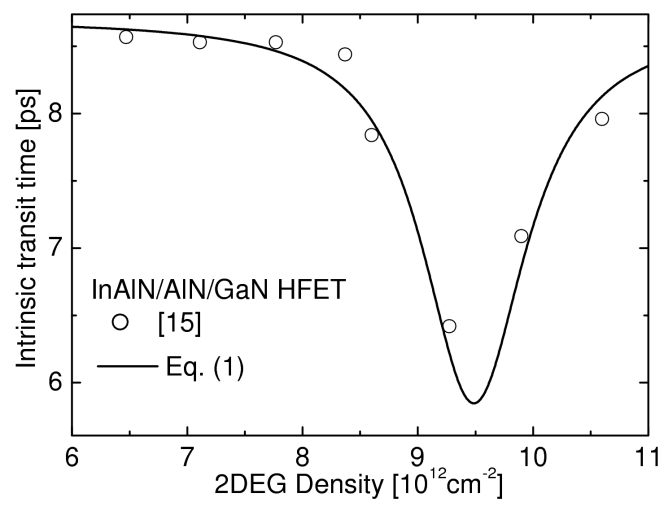

Fig. 4. Dependence of intrinsic transit time on 2DEG density for InAlN/AlN/GaN HFETs (circles [15]) and Eq. (1) for $n_{\text {res }}=9.5 \times 10^{12} \mathrm{~cm}^{-2}$ (curve).

\section{Summary}

The resonance dependence of the hot-phonon lifetime on the electron density can serve as a signature of plasmon-assisted decay of hot phonons. The signature has been resolved from independent measurements of the intrinsic signal delay time and the drain current degradation for InAlN/AlN/GaN HFETs. The conditions for the minimum negative effect of hot phonons are specified.

\section{Acknowledgments}

This work is supported by grants FA8655-09-1-3103 and FA9550-04-1-04-14 from the US Air Force Office of Scientific Research under the direction of Dr. Kitt Reinhardt.

\section{References}

[1] H. Morkoç, Handbook of Nitride Semiconductors and Devices, Vol. 3. Wiley-VCH, Weinheim 2009.

[2] J.H. Leach, H. Morkoç, Proc. IEEE 98, 1127 (2010).

[3] A. Matulionis, J. Liberis, I. Matulionienè, M. Ramonas, E. Šermukšnis, Proc. IEEE 98, 1128 (2010).

[4] A. Matulionis, Phys. Status Solidi C 6, 2834 (2009).

[5] B.K. Ridley, J. Phys., Condens. Matter 8, L511 (1996).

[6] K.T. Tsen, J.G. Kiang, D.K. Ferry, H. Morkoç, Appl. Phys. Lett. 89, 112111 (2006).

[7] A. Dyson, B.K. Ridley, J. Appl. Phys. 103, 114507 (2008).

[8] A. Matulionis, J. Liberis, I. Matulionienè, M. Ramonas, L.F. Eastman, J.R. Shealy, V. Tilak, A. Vertiatchikh, Phys. Rev. B 68, 035338 (2003).

[9] A. Matulionis, J. Liberis, I. Matulionienè, M. Ramonas, E. Sermukšnis, J.H. Leach, M. Wu, X. Ni, X. Li, H. Morkoç, Appl. Phys. Lett. 95, 192102 (2009).

[10] Z. Wang, K. Reimann, M. Woerner, T. Elsaesser, D. Hofstetter, J. Hwang, W.J. Schaff, L.F. Eastman, Phys. Rev. Lett. 94, 037403 (2005).

[11] A. Matulionis, J. Liberis, H. Morkoç, Proc. SPIE 7602, 76020H (2010).

[12] A. Matulionis, J. Liberis, I. Matulionienė, E. Šermukšnis, J.H. Leach, H. Morkoç, in: Workshop on Compound Semiconductor Devices and Integrated Circuits (WOCSDICE 2010), Darmstadt/Seeheim (Germany), 2010, p. 191.

[13] J.H. Leach, M. Wu, X. Ni, J. Lee, Ü. Özgür, H. Morkoç, J. Liberis, E. Šermukšnis, A. Matulionis, H. Cheng, Ç. Kurdak, Appl. Phys. Lett. 95, 223504 (2009).

[14] J.H. Leach, M. Wu, X. Ni, X. Li, Ü. Özgür, H. Morkoç, J. Liberis, E. Šermukšnis, A. Matulionis, H. Cheng, C. Kurdak, Y.-T. Moon, Phys. Status Solidi A 207, 1345 (2010).

[15] J.H. Leach, C.Y. Zhu, M. Wu, X. Ni, X. Li, J. Xie, Ü. Özgür, H. Morkoç, J. Liberis, E. Šermukšnis, A. Matulionis, T. Paskova, E. Preble, K.R. Evans, Appl. Phys. Lett. 96, 133505 (2010). 